\title{
The concept of model rays in domain conversion
}

Eduardo Filpo (Petrobras) and Rodrigo Portugal (Landmark | Halliburton)

Copyright 2019, SBGf - Sociedade Brasileira de Geofísica

This paper was prepared for presentation during the $16^{\text {th }}$ International Congress of the Brazilian Geophysical Society held in Rio de Janeiro, Brazil, 19-22 August 2019.

Contents of this paper were reviewed by the Technical Committee of the $16^{\text {th }}$ International Congress of the Brazilian Geophysical Society and do not necessarily represent any position of the SBGf, its officers or members. Electronic reproduction or storage of any part of this paper for commercial purposes without the written consent of the Brazilian Geophysical Society is prohibited.

\begin{abstract}
In this work, we present the concept of model rays which can be seen as the dual of image rays, but traced in time domain. We present the system of first order differential equations, which allows the numerical computation of the model rays using a velocity field in time domain. We demonstrate with a synthetic example that model rays traced with this system of equations are identical to others traced with two different methods. Among other geometrical interpretations, the model rays can be seen as the instrument to perform the time-to-depth conversion of seismic images. Time-to-depth conversion algorithms which make use of model rays are much simpler than those one based on image rays. Image rays are more suitable to perform depth-to-time conversion.
\end{abstract}

\section{Introduction}

Time migration methods are still widely used in the industry not only because they are more robust and faster than depth domain ones, but also because shifts in technology paradigms take longer to happen in the oil industry. The conversion of seismic sections from time to depth domains has been possible after the introduction of the concept of image ray (Hubral, 1977), which validity is subject to the same conditions of validity of time migration methods. Subsequently, the use of image rays was extended to the conversion of seismic images (Silva et al 2009, Filpo et al 2016) and time migration velocity models (Silva et al, 2009; Cameron et al 2007 and Iversen et al 2008).

In general, time-to-depth conversion algorithms based on image ray tracing or, equivalently, based on image wavefront construction, involve mapping of values distributed along a regular grid in the time domain into another regular grid in the depth domain. This mapping between domain is constructed with image rays traced in depth, using velocity information available in depth or either in time domain. However, no matter which approach is taken, mapping domains using image rays results in an irregular distribution of points in the depth domain. This is far from optimal because high multiplicity or voids of values can happen.

Figure 1 shows a velocity model in depth superimposed by a set of image rays and its corresponding wavefronts. This curves forms a curvilinear grid directly associated with a cartesian grid in time domain, in which seismic traces mapped into image rays and time slices into wavefronts. For that example, the velocity model follows the affine equation:

$$
V(x, z)=1.95+0.1 z+0.15 x,
$$

where $x$ and $z$ in kilometers and $V$ in kilometers per second.

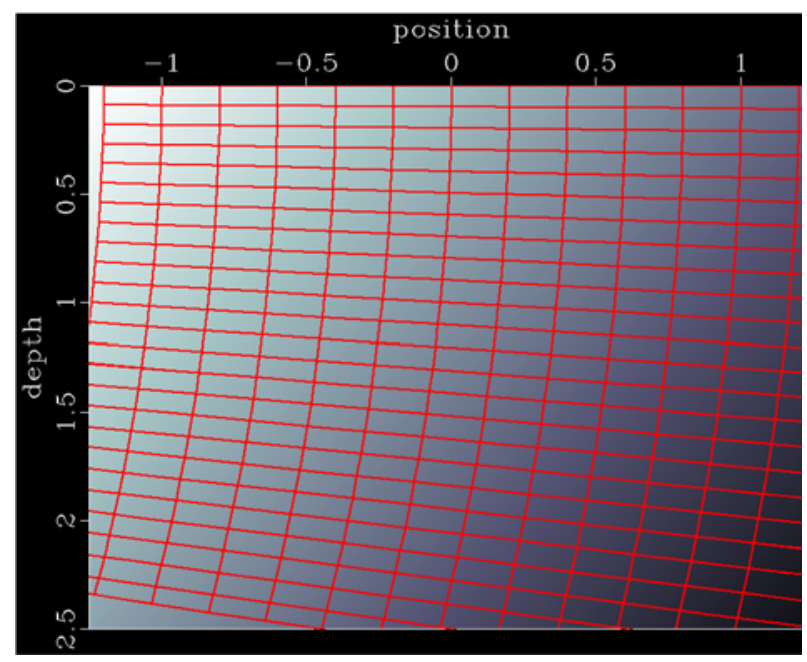

Figure 1: Velocity model in depth superimposed by a set of image rays and its corresponding wavefronts.

Filpo et al (2016) presented a solution to the problem of spreading properties in the time-depth conversion with image rays that is the use of the Taylor series. Such strategy became feasible from the formulation of time-todepth conversion as a change of coordinates problem, where vertical lines in the time domain are mapped to image rays at depth, and the horizontal lines on originally flat wave that emerge from the datum. The required derivatives to apply the Taylor series are obtained by finite differences along the wavefronts. For simplicity, we refer to this domain conversion process just as spreading algorithm. Remember that to trace image rays a velocity model in depth is required.

Figures $2 a$ and $2 b$ show an example of the spreading algorithm application. The input section is an artificial time migrated section which contains just a circular feature (time axis is upwards). The velocity model used to trace image rays and wavefronts is the same of the Figure 1 . Note that there are no caustics and neither shadow zones in this model, that is a necessary condition for the perfect working of the spreading algorithm, which is based in a coordinate transformation approach.

The same kind of trouble does not happen with the use of image rays in the conversion from depth to time, where the regular grid of output is directly filled with values collected in the depth domain through a simple interpolation. This kind of transformation is referred just as gathering 
algorithm in this text. For example, to convert a velocity model from depth to time, we just trace image rays with a desired sample rate and collect velocity values along them.
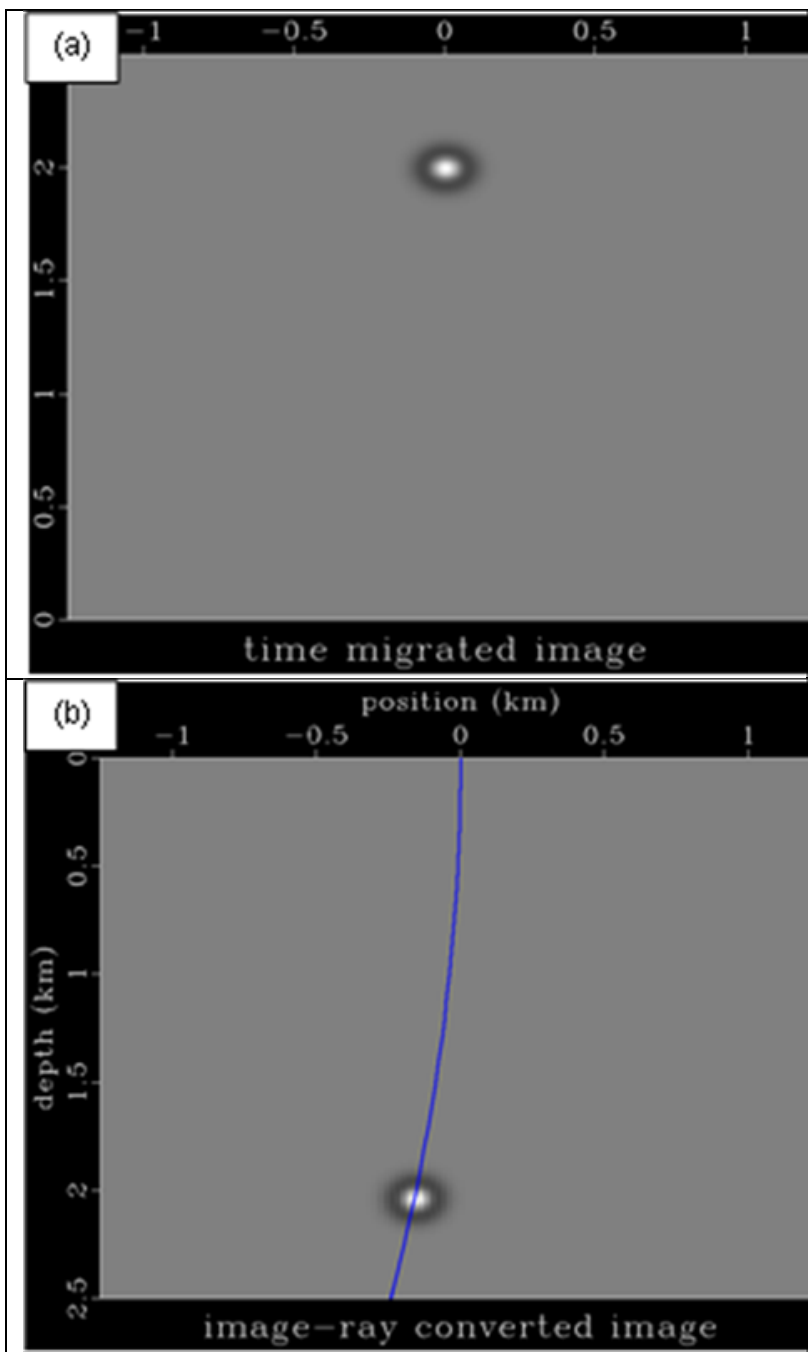

Figure 2: a) Artificial time-migrated image. b) Depthconverted image using the image-ray spreading algorithm.

The concept of model rays arose when we start to observe some features of seismic images converted from depth to time by means of a gathering algorithm, which consists of collecting amplitudes along image rays. Figure $3 a$ shows an image in depth domain with horizontal and vertical events, while Figure $3 b$ shows the time-converted image by means of the gathering algorithm. The observation of the converted section clearly shows that the vertical lines in depth are transformed into curves in the time domain with a behavior very similar to the image rays. Note that the time axis is upwards in the converted image.

The similarity between the curves defined by the converted features and the image rays instigate us to understand them and to figure out if they are rays (Silva et al, 2009). The name model rays was coined because they are associated with vertical lines in the model space (depth domain), while image rays are associated with vertical lines in the image space (time domain).
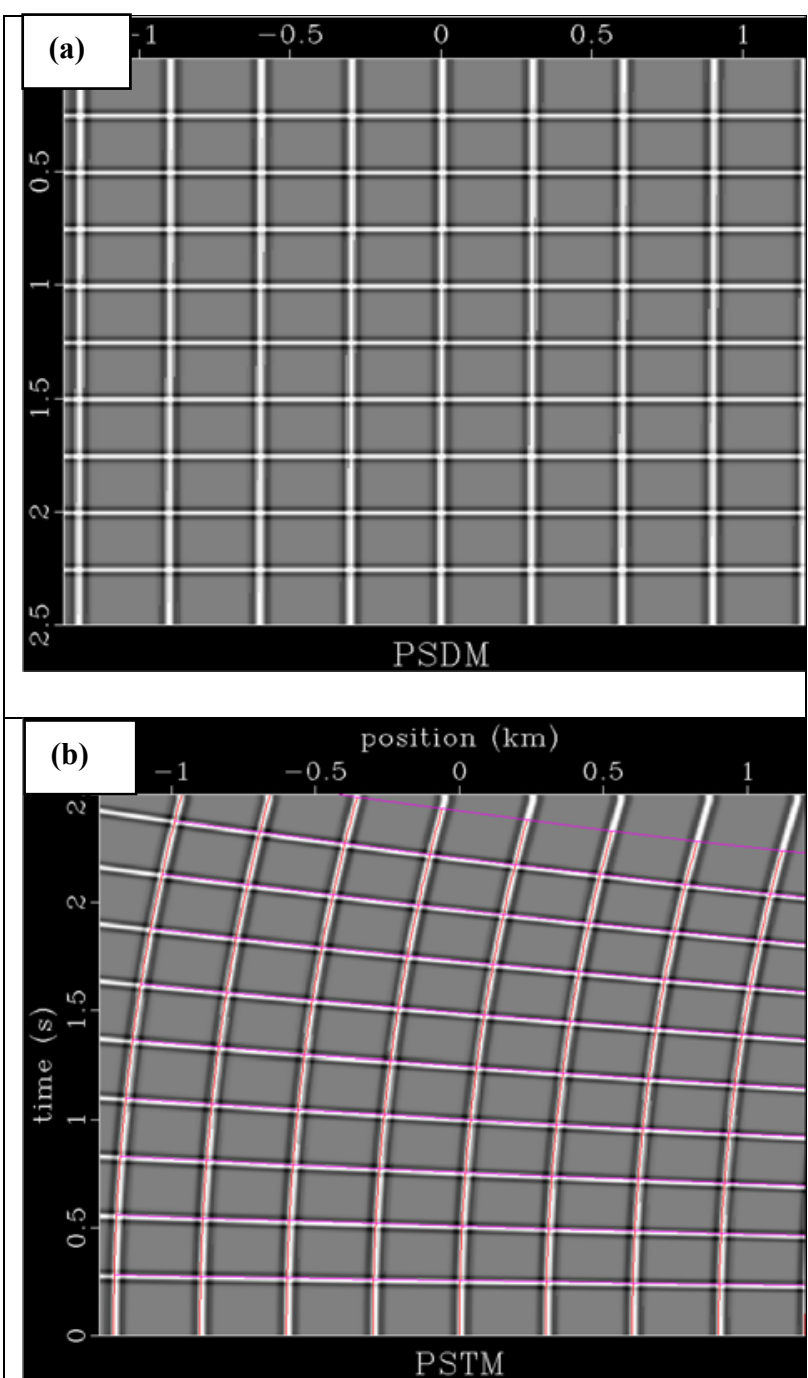

Figure 3: a) Artificial seismic image in depth. b) Timeconverted image using the image ray gathering algorithm.

\section{Derivation of ray model equations}

In this section, we present the derivation of the equations for tracing model rays in time domain. We start from two differential equations related to the conventional ray tracing theory. The first one is the well-known eikonal equation

$$
\left(\frac{\partial \tau}{\partial x}\right)^{2}+\left(\frac{\partial \tau}{\partial z}\right)^{2}=\frac{1}{V^{2}}
$$

and the second is its associated equation

$$
\left(\frac{\partial x}{\partial \tau}\right)^{2}+\left(\frac{\partial z}{\partial \tau}\right)^{2}=V^{2}
$$

hereby referred as Huygens equation, due to its use in the Huygens wavefront tracing algorithm (Sava and Fomel, 2001). In both equations, $x$ and $z$ are spatial coordinates, $\tau$ is traveltime and $V$ is interval velocity. 
The first step to derive the model ray equations consists in representing both image ray and model ray as complex variable functions, given respectively by

$$
\begin{aligned}
& F=x(\xi, s)+i z(\xi, s) \\
& G=\xi(x, z)+i s(x, z)
\end{aligned}
$$

In this representation, $\xi$ is the horizontal coordinate of the image ray at the starting point, and $s$ is the arc length along the image ray. The relationship between the arc length and the traveltime is given by the integral

$$
s=\int_{0}^{\tau} V d \tau
$$

or, alternatively, by the differential equation

$$
d s=V d \tau .
$$

By assuming that the velocity model is smooth without strong lateral velocity variations, we can impose the condition that $F$ and $G$ are differentiable in its respective domains. This assumption permits the use of CauchyRiemann equations (see the Appendix) to make a domain transformation in the original eikonal and Huygens equations.

Applying Cauchy-Riemann equations in $G$ and $F$ gives respectively

$$
V \frac{\partial \tau}{\partial x}=\frac{\partial s}{\partial x}=-\frac{\partial \xi}{\partial z}
$$

and

$$
\frac{1}{V} \frac{\partial x}{\partial \tau}=\frac{\partial x}{\partial s}=-\frac{\partial z}{\partial \xi}
$$

Substituting (5) into the eikonal equation (1) leads to

$$
\left(\frac{\partial \xi}{\partial z}\right)^{2}+V^{2}\left(\frac{\partial \tau}{\partial z}\right)^{2}=1
$$

which is a Huygens-type equation, but in time domain. On the other hand, substituting (6) into Huygens equation (2), gives

$$
\left(\frac{\partial z}{\partial \xi}\right)^{2}+\frac{1}{V^{2}}\left(\frac{\partial z}{\partial \tau}\right)^{2}=1
$$

which corresponds to an eikonal-type equation, but in time domain. In this equation, $z=z(\xi, \tau)$ plays the role of pseudo-wavefront in time domain. Zero-order ray theory says that the rays are the base characteristic curves of an eikonal equation.

The next step to derive the model ray tracing system is to write the time eikonal equation (8) in the form of a Hamiltonian $H$ defined by

$$
H(\xi, \boldsymbol{q})=\frac{1}{2}\left[q_{\xi}^{2}+\frac{1}{v^{2}} q_{\tau}^{2}-1\right]
$$

where the vectors $\xi$ and $\boldsymbol{q}$ are respectfully the ray model position and the ray model slowness vector, in time domain, which are defined by

$$
\xi=(\xi, \tau) \text { and } \boldsymbol{q}=\left(q_{\xi}, q_{\tau}\right)=\left(\frac{\partial z}{\partial \xi}, \frac{\partial z}{\partial \tau}\right) \text {. }
$$

According to Cerveny (2001), given any Hamiltonian, a solution can be found using the method of characteristics, which are the solutions of the system of first-order differential equations

$$
\left\{\begin{array}{l}
\frac{d \boldsymbol{x}}{d s}=\nabla_{p} H \\
\frac{d \boldsymbol{p}}{d s}=-\nabla_{x} H \\
\frac{d t}{d s}=\boldsymbol{p}^{T} \nabla_{p} H
\end{array}\right.
$$

where $H$ is a real valued function, called the Hamiltonian, $\boldsymbol{p}=\left(p_{x}, p_{y}\right)$ is the slowness vector, $\boldsymbol{x}=(x, y)$ is the position vector in time domain, $t$ is the traveltime and $s$ is an integration parameter. The initial conditions for any ray is that it starts at given point $\boldsymbol{x}(0)=\boldsymbol{x}_{0}$ with any given nonzero slowness $\boldsymbol{p}(0)=\boldsymbol{p}_{0}$ at the initial time of propagation $t(0)=t_{0}$.

Bu using the Hamiltonian (8) in the ray equations (1), we obtain the model ray equations

$$
\left\{\begin{array}{l}
\frac{d q_{\xi}}{d z}=\frac{1}{v^{3}} \frac{\partial v}{\partial \xi} q_{\xi}^{2} \\
\frac{d q_{\tau}}{d z}=\frac{1}{v^{3}} \frac{\partial v}{\partial \tau} q_{\tau}^{2} \\
\frac{d \tau}{d z}=\frac{1}{v^{2}} q_{\tau} \\
\frac{d \xi}{d z}=q_{\xi}
\end{array}\right.
$$

where the traveltime $\tau$ and $\xi$ plays the role of vertical and horizontal coordinates in time domain and $z$ (depth) works as an integration parameter. The initial conditions for any model ray is that it starts at given point at the measurement surface $\xi(0)=\xi_{0}$ with a vertical slowness $\boldsymbol{q}(0)=\boldsymbol{q}_{0}$ at the initial depth of propagation $z=0$.

As we saw earlier, the inverse transformation from depth to time can be easily performed from gathering amplitudes along image rays. In the same way, time-to-depth conversion can be carried out by means of collecting values along model rays. Simply speaking, algorithms involving collection of values are generally much simpler than those involving scattering. Another advantage of using model rays is that they require velocity field in time domain, which can be obtained directly from time-migration velocity analysis.

\section{Numerical experiment}

Figure 4 summarizes the results of a simple experiment designed to validate the system of model ray equations. The idea is to compare the trajectory of a model ray traced by solving the system (11), with other two different methods. The first method tracks the model ray trajectory by tracing several image rays, whose initial conditions are defined by paraxial ray theory. The second method uses a finite-difference algorithm to solve a Huygens type equation to trace pseudo wavefronts and its orthogonal lines, which corresponds to model rays. Both methods are described in Silva et al, 2009. Note that there are no significant differences between the three plotted curves. It is possible to observe that exist more than one curve just because they are plotted with different line widths. 
The first step to solve the model ray tracing system is to create a velocity field in time domain. In this case, we just convert from depth to time the velocity model of Figure 1 with the image ray collecting algorithm. Then, the model rays are numerically traced, step by step, solving the system (11) by using the fourth order Runge Kutta method.

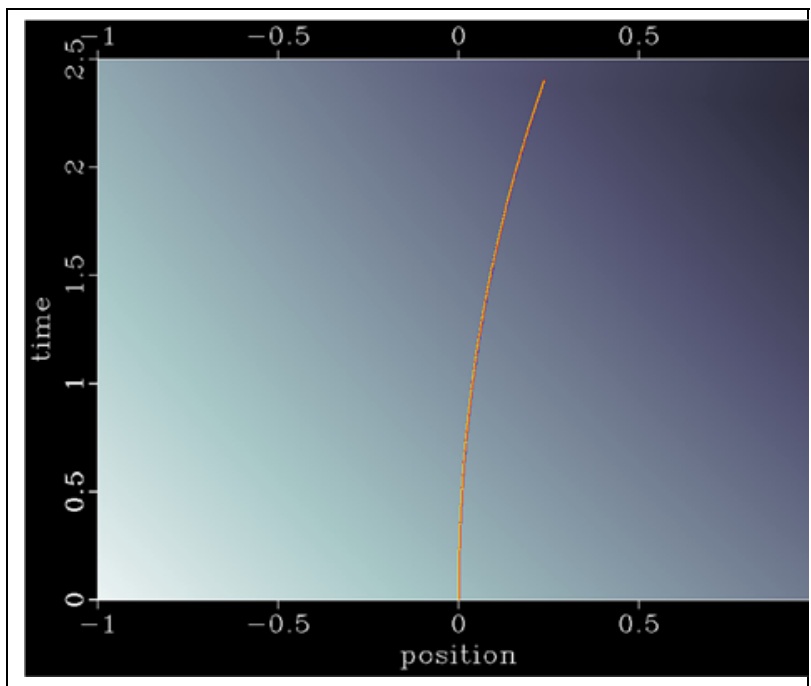

Figure 4: Velocity model in time superimposed by three model rays traced with different methods: 1) two-point image rays tracing, the wider line plotted in blue, 2) wavefront construction using Huygens principle, regular line plotted in red, and 3) the Runge Kutta method, the thinner line plotted in yellow. Note that time axis is upwards.

\section{Conclusions}

In this paper, we derived a differential system for tracing model rays in time domain, by starting from a first-order non-linear partial differential equation in time and applying the zero-order ray theory. We validate positively this system of equations by comparing the computed ray model trajectories with others obtained with two different methods. Note that, for the sake of simplicity, we derived just the equations for $2 \mathrm{D}$ data, but there is no restrictions or special conditions for the derivation in the 3D case, and its extension is straightforward.

We also demonstrate that model rays are more suitable than image rays to perform time-to-depth conversion, because it permits the use of gathering algorithms, which are much simpler than the spreading types. With such approach, the amplitudes in time can be uniquely transferred to their converted position in depth.

Additionally, another advantage of using of model rays to carry out time-to-depth conversion is that they make use of velocity field in time, which can be obtained directly from migration velocity analysis (MVA) applied in time migration processes.

\section{Acknowledgments}

The authors would like to thank Petrobras and Halliburton for the support.

\section{References}

Cameron, M., S. Fomel, and J. Sethian, 2008, Time-todepth conversion and seismic velocity estimation using time migration velocity. Geophysics, 73(5), VE205-VE210. doi: 10.1190/1.2967501.

Červený, V., 2001, Seismic ray theory: Cambridge University Press.

Iversen, E. and Tygel, M., 2008, Image-ray tracing for joint 3D seismic velocity estimation and time-to-depth conversion. Geophysics, 73(3), S99-S114.

Hubral, P., 1977, Time migration: some ray theoretical aspects: Geophysical Prospecting, 25, 738-745. doi: 10.1111/j.1365-2478.1977.tb01200

Filpo, E., R. Portugal, N. Zago, P. M. Cunha, A. Vicentini, and J. L. Carbonesi, 2016, Image-ray concept as the key to 20 years of success of time-to-depth conversion in Petrobras: The Leading Edge, 35, 323-328, doi: 10.1190/tle35040323.1

Sava, P and Fomel S., 2001, 3-D traveltime computation using Huygens wavefront tracing. Geophysics, 66(3), 883889.

Silva, E. F. F., Portugal, R., and Vicentini, A., 2009, Model rays for depth-to-time conversion. In 11th International Congress of the Brazilian Geophysical Society \& EXPOGEF 2009. doi: 10.1190/sbgf2009-213.

\section{Appendix}

Cauchy-Riemann Theorem: a necessary and sufficient condition for a complex function

$$
F(x+i y)=u(x, y)+i v(x, y)
$$

to be holomorphic (complex differentiable) is that the following equations are valid

$$
\begin{aligned}
& \frac{\partial u}{\partial x}=\frac{\partial v}{\partial y} \\
& \frac{\partial u}{\partial y}=-\frac{\partial v}{\partial x}
\end{aligned}
$$

These are known as the Cauchy-Riemann equations. 